\title{
Patrimonialisation et territorialisation de la littérature : causes, enjeux et effets
}

Patrimonialization and Territorialization of Literature: Causes, Implications and Effects

\section{Marie-Françoise Melmoux-Montaubin}

\section{OpenEdition}

\section{Journals}

Édition électronique

URL : https://journals.openedition.org/recherchestravaux/2361

DOI : 10.4000/recherchestravaux.2361

ISSN : 1969-6434

Éditeur

UGA Éditions/Université Grenoble Alpes

Édition imprimée

ISBN : 978-2-37747-197-3

ISSN : 0151-1874

\section{Référence électronique}

Marie-Françoise Melmoux-Montaubin, «Patrimonialisation et territorialisation de la littérature : causes, enjeux et effets », Recherches \& Travaux [En ligne], 96 | 2020, mis en ligne le 23 juin 2020, consulté le 30 juin 2021. URL : http://journals.openedition.org/recherchestravaux/2361 ; DOI : https://doi.org/ 10.4000/recherchestravaux.2361

Ce document a été généré automatiquement le 30 juin 2021.

(C) Recherches \& Travaux 


\title{
Patrimonialisation et territorialisation de la littérature : causes, enjeux et effets
}

Patrimonialization and Territorialization of Literature: Causes, Implications and Effects

\author{
Marie-Françoise Melmoux-Montaubin
}

\section{Le « patrimoine littéraire », objet nouveau mal identifié}

\section{Une utilisation récente dans le monde du livre et de la littérature}

1 La patrimonialisation de la littérature, relativement récente, adopte différentes formes, de la patrimonialisation du livre comme objet à celle du texte. Dans le monde du livre, le passage des «fonds anciens » au « patrimoine » s'est mis en place autour du rapport Desgraves de $1982^{1}$ :

[...] les bibliothèques ont d'abord possédé des manuscrits et imprimés dont elles dressèrent lentement les catalogues ; plus tard, elles se découvrirent des "trésors " dont elles publièrent des listes choisies ; plus récemment, elles distinguèrent [...] des fonds anciens, qui devinrent assez vite «anciens, rares et précieux» [...]. Et, voici quinze ans à peine, elles se donnèrent la charge lourde et magnifique d'un patrimoine, d'un bien collectif, dont elles se sentaient comptables devant les générations futures mais aussi devant la collectivité actuelle ${ }^{2}$.

Les fonds patrimoniaux ne concernent pas exclusivement le livre, puisqu'on y trouve différents objets, manuscrits, pièces de monnaies, sceaux, dessins, etc. ${ }^{3}$; leur dimension n'est pas essentiellement « littéraire », puisque le « beau » livre, le livre ancien, le livre rare, sont privilégiés, leur contenu ou leur qualité venant en quelque sorte par surcroît.

C'est bien en revanche la littérature en tant que telle qui est inscrite au patrimoine avec l'introduction $\mathrm{du}$ socle commun des connaissances dans les programmes scolaires : 
Il est tout à fait notable que les expressions "patrimoine littéraire ", « littérature patrimoniale ", "œuvres du patrimoine", «textes patrimoniaux», etc. vont intégrer l'ensemble des programmes parus entre 2008 et 2012, de la maternelle au lycée. Ce phénomène est d'autant plus remarquable que le terme "patrimoine " était totalement absent des programmes précédents, lesquels, parlant des mêmes œuvres, les qualifiaient de « majeures », « de référence » ou « significatives ». [...] Je considère donc $[. .$.$] que le patrimoine littéraire est un objet scolaire nouveau { }^{4}$. essentiellement développée dans le domaine des sciences humaines et sociales, a gagné la recherche universitaire en lettres: un nombre non négligeable d'équipes de recherche en littérature se place sous ce titre, le terme de patrimoine étant le plus souvent associé à d'autres : « Patrimoine, Littérature, Histoire » (université de Toulouse Jean Jaurès), «Centre interdisciplinaire de recherche sur les patrimoines en lettres et langues» (université d'Angers), "Culture, patrimoine et sociétés numériques » (université d'Avignon), «Dynamiques patrimoniales et culturelles» (université de Versailles-Saint-Quentin), "Patrimoines, création et transmission des savoirs, éducation, humanités» (université de Cergy-Pontoise). Lors même que le " patrimoine » ne figure pas dans leur nom, certains laboratoires l'inscrivent au cœur de leur projet, comme par exemple l'équipe CORPUS des anglicistes de l'université de Picardie Jules Verne qui a organisé son dossier de contractualisation autour de la notion de "Patrimoine : rupture et réappropriation », la notion de patrimoine étant " appliquée aux civilisations et aux littératures du monde anglophone ».

Plusieurs projets de recherche ont été récemment déposés autour de cette question. Je n'en mentionnerai que deux, plus spécifiquement articulés autour de questionnements littéraires : La Fabrique du patrimoine littéraire: les collections de monographies illustrées de poche consacrées aux écrivains (1944-2015), projet porté par les universités de Louvain, Laval, Paris 4 Sorbonne et Lausanne ; ou encore le projet LIMECO (Littérature et mémoire collective: Comprendre et visualiser l'ancrage du littéraire dans un territoire), porté par l'université de Nantes et financé par le contrat de plan État-région: il est présenté comme "une étude des modes de patrimonialisation de la littérature", qui vise "à mettre au jour les logiques d'ancrage du littéraire dans des lieux ${ }^{5} »$.

Le succès de l'idée de "patrimonialisation » étant désormais avéré en ce qui concerne les textes, il devient nécessaire non seulement d'en comprendre les raisons, mais encore d'essayer d'en saisir les enjeux et, si possible, les effets sur la littérature. Quatre éléments au moins expliquent la faveur dont elle jouit : l'indétermination du concept, qui permet de l'appliquer à quelque domaine que ce soit; son caractère transdisciplinaire, dans l'air du temps; sa dimension essentiellement consensuelle; enfin les perspectives économiques et commerciales qu'il ouvre.

\section{Un concept flou}

7 La notion de patrimoine est difficile à cerner, comme en témoigne l'inflation des articles et ouvrages publiés autour de cette question depuis les années $1980^{6}$ :

[...] le patrimoine, au sens où on l'entend aujourd'hui dans le langage officiel et dans l'usage commun, est une notion toute récente, qui couvre de façon nécessairement vague, tous les biens, tous les « trésors » du passé7.

8 Si le patrimoine a d'abord eu un sens très précis, désignant l'« ensemble des biens hérités des ascendants ou réunis et conservés pour être transmis aux descendants ${ }^{8} »$, sa 
définition s'est en effet considérablement élargie après la Révolution française: l'inventaire des biens du clergé et de la noblesse réalisé à partir de février 1794 par l'abbé Grégoire rassemble les monuments, abbayes, églises, châteaux et les œuvres qu'ils contiennent au titre de leur valeur historique et esthétique et de leur intérêt pour la nation. Le patrimoine, alors strictement sélectionné ${ }^{9}$, apparaît comme constitutif de l'identité nationale. Il s'est depuis quelques années ouvert au "patrimoine naturel» ou au "patrimoine culturel immatériel», trouvant une extension maximale qui suscite de nombreuses réflexions, le plus souvent critiques, de Nathalie Heinich évoquant l'assomption de la " petite cuillère ${ }^{10}$ » à Alain Babadzan soulignant la "frénésie patrimoniale ${ }^{11}$ ", tandis que d'autres mettent en évidence $L a$ Machinerie patrimoniale ${ }^{12}$. «Si tous les auteurs constatent que le corpus patrimonial est le fruit d'un choix, sanctionné par des institutions légitimes et légitimantes, certains voient davantage dans la diversité du patrimoine de la fin du $\mathrm{xx}^{\mathrm{e}}$ siècle un non-choix: tout est patrimoine, ou presque ${ }^{13}$.» On peut alors parler de folie de la patrimonialisation ou, selon la suggestion de Henri-Pierre Jeudy, de " patrimoines en folie ${ }^{14} »$. Le passage du singulier au pluriel témoigne à sa manière de cette évolution.

\section{Patrimoine et transdisciplinarité}

9 La fortune du terme procède également de sa capacité à s'articuler aussi bien avec l'histoire qu'avec la culture entendue en son sens le plus large, aussi bien avec les savoirs qu'avec les humanités. Le "patrimoine» fonctionne ainsi comme un indice d'inter ou de transdisciplinarité, la transdisciplinarité se définissant comme ce qui fait "émerger une nouvelle discipline ${ }^{15}$ »: les "sciences du patrimoine ${ }^{16}$ ", convoquant historiens, anthropologues, ethnographes, géographes, philosophes, littéraires (la liste pourrait sans doute être plus longue) relèvent de cette transdisciplinarité fort prisée dans une sphère intellectuelle qui ne redoute rien tant que l'enfermement des disciplines sur elles-mêmes et une spécialisation comprise comme réduction du champ.

\section{Un objet consensuel}

10 Il est encore une autre raison qui explique que la patrimonialisation bénéficie d'un fort coefficient de sympathie a priori: dans une société « mondialisée » ou "globalisée », elle apparaît comme défense nécessaire de la (ou des) diversité(s). D'abord comprise comme une démarche de détection/conservation/valorisation des biens qui constituent l'identité d'un groupe donné, la patrimonialisation a su évoluer et se faire reconnaître comme lutte contre l'uniformisation et mise en valeur des diversités. La définition du «patrimoine culturel immatériel » sur le site de l'UNESCO est à cet égard édifiante et souligne particulièrement clairement la tension entre identité et diversité, la tentation identitaire inhérente à la patrimonialisation étant en quelque sorte rachetée par l'idée du maintien de la diversité contre la globalisation:

Le patrimoine culturel ne s'arrête pas aux monuments et aux collections d'objets. Il comprend également les traditions ou les expressions vivantes héritées de nos ancêtres et transmises à nos descendants, comme les traditions orales, les arts du spectacle, les pratiques sociales, rituels et événements festifs, les connaissances et pratiques concernant la nature et l'univers ou les connaissances et le savoir-faire nécessaires à l'artisanat traditionnel.

Bien que fragile, le patrimoine culturel immatériel est un facteur important du maintien de la diversité culturelle face à la mondialisation croissante ${ }^{17}$. 
... ou comment glisser en l'espace de quelques lignes des «traditions [...] héritées de nos ancêtres ", dont la dimension identitaire est évidente quoique passée sous silence, à la lutte contre la globalisation.

\section{Perspectives économiques et commerciales}

12 La patrimonialisation est aussi une réalité économique : défini comme bien inaliénable et donc a priori étranger aux circuits économiques, le patrimoine est néanmoins une richesse monnayable, en raison notamment de l'intérêt touristique attaché aux lieux de patrimoine quels qu'ils soient: l'empressement des différents territoires à faire reconnaître leurs atouts patrimoniaux en témoigne sans détour ${ }^{18}$.

13 C'est dans ce contexte que s'est développée ces dernières années l'inscription de la littérature dans une perspective patrimoniale et c'est à l'aune de ces éléments qu'il faut la lire. Il s'agira donc moins de parler ici du « patrimoine littéraire » comme d'un objet plus ou moins clairement défini que de la patrimonialisation de la littérature et des conséquences de ce processus.

\section{La patrimonialisation : un grand récit}

Si le patrimoine est difficile à définir et ses contours flous, il n'en va pas de même pour la patrimonialisation. Elle est un processus concret pour lequel Guy Di Méo reconnaît " six étapes successives et enchaînées les unes aux autres. Elles vont de la prise de conscience patrimoniale à la valorisation du patrimoine, en passant par les phases essentielles de sa sélection et de sa justification, de sa conservation et de son exposition $^{19}$."

15 À l'origine du processus comme à son terme, se trouve donc un récit ${ }^{20}$. Inscrire au patrimoine, c'est faire reconnaître en quelque chose (un monument, un livre, un mode de vie, un paysage) un élément constitutif d'une communauté ; c'est donc écrire l'histoire de cette communauté. La patrimonialisation, geste politique au sens fort, fonde le groupe : elle dit moins ce qu'il est que ce qu'il veut être, ce en quoi il veut se reconnaître. À bien des égards, le geste de patrimonialisation vaut comme récit ou mythe des origines. Le récit se trouve aussi à l'autre bout de la chaîne, dans les opérations de valorisation de l'objet "patrimonialisé ", qui consistent à raconter en quoi il compte, ce qu'il dit, afin de rassembler le plus efficacement possible la communauté autour de lui.

16 La patrimonialisation en tant que récit - peut-être même plus encore en tant que fiction - a donc à voir de manière essentielle avec la littérature. Elle échoue cependant, me semble-t-il, à être le grand récit des origines qu'elle pourrait prétendre incarner, et sa faillite en la matière tient à la nature même du processus : est inscrit au patrimoine, matériel ou immatériel, ce qui est en danger ${ }^{21}$. Le récit que porte la patrimonialisation est donc par essence catastrophiste.

\section{Patrimonialisation et catastrophisme}

17 Le déploiement de l'idée de " patrimoine littéraire » peut ainsi légitimement apparaître comme l'indice d'une crise de la littérature, considérée comme menacée de disparition, 
«affectée par ce sentiment de perte et d'effacement propre au patrimoine ${ }^{22}$ ». Ce n'est sans doute pas un hasard si, en France, l'idée de patrimonialisation des textes s'est imposée dans les bibliothèques puis dans les programmes scolaires dans les dernières décennies $\mathrm{du} \mathrm{xx}^{\mathrm{e}}$ siècle, à une période où se multiplient les analyses sur le déclin de la culture et la fin d'une certaine exception française. Est-ce même un hasard si l'idée de "patrimoine littéraire" intègre "l'ensemble des programmes parus entre 2008 et $2012^{23}$ ", deux ans après la charge de Nicolas Sarkozy, alors candidat à l'élection présidentielle, contre La Princesse de Clèves, plus précisément contre l'inscription de ce texte au concours d'attaché d'administration? C'est quand un candidat à l'élection présidentielle prend acte de l'absence de pertinence de la culture littéraire dans la vie courante ou quotidienne que les programmes scolaires adoptent le lexique de la patrimonialisation.

18 Plus encore : inscrite au patrimoine, la littérature rejoint châteaux, églises et parcs, mais aussi bien la bière belge, inscrite depuis 2016 au "patrimoine immatériel de l'humanité ", ou bientôt le camembert au lait cru, dont les fervents dégustateurs ont entamé les démarches en ce sens ${ }^{24}$. Ainsi, «[d]éfinie comme relevant du patrimoine culturel, la littérature est considérée comme un objet culturel comme les autres. C'est la continuation logique d'un affaiblissement en termes de positionnement hiérarchique et symbolique tant dans le champ social que dans le champ de l'enseignement. Il y a un brouillage des spécificités : les œuvres sont considérées comme des objets culturels et la lecture des œuvres devient une pratique culturelle parmi d'autres ${ }^{25}$.» Sortie de sa "tour d'ivoire" pour être plongée dans le "patrimoine" dont elle constitue un élément parmi d'autres, que devient la littérature? Pour formuler les choses autrement, la littérature est-elle soluble dans le patrimoine?

\section{Patrimonialisation et territorialisation}

\section{Une géographie littéraire}

C'est l'une des questions que pose le développement des maisons d'écrivains, indice important, même s'il n'est évidemment pas le seul, de la patrimonialisation de la littérature. Si la Fédération nationale des maisons d'écrivain et des patrimoines littéraires est née à la fin des années $1990^{26}$, structurant un réseau qui ne demandait qu'à se développer, les écrivains étaient depuis longtemps déjà inscrits «au patrimoine » de diverses manières : statues, monuments, noms de rues, d'écoles, etc. La maison d'écrivain possède cependant une spécificité, celle d'inscrire l'écrivain dans un territoire, considéré comme "son" territoire: si nombre d'institutions scolaires, notamment d'écoles primaires, portent le nom de Jules Verne ${ }^{27}$, seule la ville d'Amiens qu'il avait élue comme son lieu de vie et d'activité possède une «Maison de Jules Verne », Nantes, sa ville natale, offrant de son côté un « Musée Jules Verne ».

Cette territorialisation n'est pas sans conséquences sur les études littéraires, à moins qu'elle n'ait été l'un des moteurs de leur évolution. Christine Baron souligne ainsi un changement dans « l'histoire récente des études littéraires » :

[...] marquées pendant le dernier tiers du $\mathrm{xx}^{\mathrm{e}}$ siècle par le déconstructionnisme, elles se sont attachées à montrer le conflit entre littérature et représentation, et à ce titre, ont défendu une conception de la littérature déterritorialisée. [...] Un intérêt renouvelé pour des notions communes avec la géographie (activités 
humaines, réalités politico-économiques, paysages et réalités sociales) montre indéniablement un changement de perspective ${ }^{28}$.

Réfléchissant sur ce renouvellement, Michel Collot distingue la "géographie de la littérature » ou « géographie littéraire » de la géocritique et la géopoétique.

Je propose d'appeler géocritique l'analyse des représentations littéraires de l'espace telle qu'on peut la tirer d'une étude du texte ou de l'œuvre d'un auteur, et non plus de son contexte. Il s'agit pour elle d'étudier moins les référents ou les références dont s'inspire le texte que les images et les significations qu'il produit, non pas une géographie réelle mais une géographie plus ou moins imaginaire. [...] Le terme de géopoétique [...] semble susceptible de désigner à la fois une poétique : une étude des formes littéraires qui façonnent l'image des lieux, et une poiétique: une réflexion sur les liens qui unissent la création littéraire à l'espace ${ }^{29}$.

Les maisons d'écrivains, quels que soient leurs efforts et leur souci critique ${ }^{30}$, s'inscrivent essentiellement dans la " géographie de la littérature » qui «montre bien comment une œuvre s'ancre dans un territoire, mais [...] oublie de montrer comment elle le transforme pour construire son propre espace, qui est celui de l'imaginaire et de l'écriture, qu'on ne trouve que dans le texte, et qu'on ne peut reporter sur aucune carte du monde connu ${ }^{31} »$. Le biais est structurel : destinée à valoriser l'ancrage d'un écrivain dans une ville, une région, un paysage, la Maison est vouée à insister d'abord sur cette attache.

Conçue comme un lieu "où souffle l'esprit ${ }^{32}$ ", elle apparaît comme le vecteur d'une forme de "sacre de l'écrivain». Présupposant la "relation séminale du lieu et de l'œuvre ${ }^{33}$ ", elle ouvre aux visiteurs « le cadre dans lequel est advenu ce qui a été défini comme le "mystère" de l'écriture ${ }^{34}$. » Qu'il s'agisse du lieu de naissance de l'écrivain, de celui où il a écrit son œuvre, parfois de celui dans lequel il s'est retiré en fin de vie, la Maison suppose comme une sorte d'évidence le lien entre le lieu, l'homme et l'œuvre. Le propos est cependant ambigu. Si le lieu, dans sa singularité et son authenticité présumée, permet - c'est du moins son ambition- d'accéder à une meilleure connaissance de l'écrivain, et, par-delà l'homme, de son œuvre, la figure de l'écrivain, son nom, garantissent en retour l'intérêt du lieu. Utilisés comme une sorte de "marque ", ils deviennent occasion de valorisation du territoire supposé porter leur reconnaissance. L'œuvre est-elle dans ce processus valorisée ou valorisante ? Tout est affaire sans doute de mesure, comme le montre Delphine Saurier analysant l'histoire de la Maison de tante Léonie :

[...] le contexte des politiques patrimoniales [...] a pourtant un effet conséquent, celui de faire de la figure de Proust un instrument de valorisation du lieu historique. Se réalise par là une inversion des rôles entre écrivain et lieu : au temps de Larcher, la conservation des lieux émanait d'une volonté de faire découvrir ou reconnaître Proust [...]; tandis qu'au temps de Borrel, c'est la figure de l'écrivain qui garantit l'existence du lieu, en tant qu'auteur désormais reconnu, dont l'image semble vivre par elle-même ${ }^{35}[. .$.$] .$

Delphine Saurier poursuit :

[...] la figure de Proust s'est vue happée par des univers bien différents jusqu'à son épanouissement dans une large sphère privée de lecteurs. C'est ainsi qu'elle vit par elle-même, en dehors du lieu historique : elle est devenue patrimoine universel. Aussi, si la Maison de tante Léonie reste une médiation pour la figure de l'écrivain, elle l'est dans une moindre mesure; alors que la figure de Proust est devenue la médiation vitale pour le lieu historique, sa reconnaissance internationale justifie la venue des publics et, par voie de conséquence, la patrimonialisation du lieu ${ }^{36}$. les traces de tel ou tel écrivain, Flaubert aux alentours de Rouen, Barbey d'Aurevilly 
dans le Cotentin, Hector Malot dans le Val de Nièvre, etc. Un simple regard sur la page «Patrimoine littéraire lorrain » du site Internet « La Lorraine des écrivains » porté par l'université de Lorraine témoigne de cette ambivalence: «le patrimoine littéraire en tant qu'espace d'identification, d'appropriation, s'avère être une ressource particulièrement intéressante pour le développement territorial et culturel de la Lorraine ", précise le chapeau, avant d'inviter à un voyage sur les routes lorraines " autour de trois formes spécifiques (maison d'écrivain, musée, balade littéraire) ${ }^{37}$. " La patrimonialisation fait ainsi de la littérature une ressource touristique et économique.

Elle opère, surtout, un changement de paradigme qui concourt à détrôner l'histoire littéraire au profit d'une approche territoriale :

Cette localisation de plus en plus intense rejoint désormais, en France, le mouvement irréversible de décentralisation du pouvoir. Les entités nouvelles ou récemment confirmées (régions, départements) travaillent à faire exister symboliquement leur territoire. Elles utilisent pour cela l'histoire et, à l'occasion, le culte des grands hommes et des hauts lieux, l'écrivain en fait partie et lentement la géographie régionale de la littérature commence, çà et là, à prendre le pas sur son histoire nationale ${ }^{38}$.

\section{« L'empaysement » contre l'universalité ?}

On ne saurait négliger l'effet de cette territorialisation sur la réception de l'écrivain qui se trouve ainsi étroitement attaché à un territoire, "empaysé » pour reprendre l'expression de Jean-Christophe Bailly ${ }^{39}$, quelle que soit la réalité du lien que son œuvre entretient avec le territoire : si Barrès, dont on connaît le culte des racines et l'importance qu'il accorde à la terre, au sol natal, est étiqueté Lorrain, de même Jules Verne peut-il être Nantais ou Amiénois par privilège de naissance ou d'habitation, lui dont l'œuvre se veut pourtant résolument "mondiale » voire "mondialiste », ne traitant jamais ou presque de Nantes ou d'Amiens ${ }^{40}$. La démarche patrimoniale insiste sur le « capital d'autochtonie ${ }^{41}$ » d'un écrivain.

Elle va ainsi à l'encontre du processus d'universalisation qui était celui de l'histoire littéraire: les "grands auteurs» ou les "classiques» valaient précisément par l'universalité qu'on trouvait en eux ; singuliers, ils portaient aussi un ensemble de traits susceptibles d'être valorisés en tout lieu et en tout temps. Le classique est par définition à la fois passé et présent, de tout lieu et de tout temps, capable de parler aux générations futures comme aux hommes les plus éloignés de son pays d'origine. C'est cette universalité que rompt, me semble-t-il, le processus de "patrimonialisation », qui tend tout au contraire à inscrire précisément un écrivain ou un texte dans un lieu et dans un temps.

\section{Patrimonialisation et légitimation}

La patrimonialisation s'opposerait à cet égard à la consécration ou à la légitimation, au processus par lequel un écrivain devient un "classique ». Parle-t-on de la même chose quand on parle des « classiques » et de la " littérature patrimoniale »? Les programmes scolaires semblent confondre les deux termes, les « auteurs du patrimoine » recouvrant désormais ceux que des programmes plus anciens désignaient comme classiques. Certains critiques passent de même d'un terme à l'autre, sans paraître faire de différence entre les deux: c'est ce que fait par exemple Christiane Chaulet-Achour, 
définissant le "patrimoine " comme "des œuvres qui ont déjà acquis patine et légitimité [...] corpus littéraire [...] circonscrit aux [...] "classiques" ${ }^{2} »$, les guillemets qui entourent et soulignent l'épithète "classiques » pouvant apparaître comme une marque de distance vis-à-vis d'un terme emprunté à d'anciennes rhétoriques.

Il existe cependant une différence entre les processus de création des "classiques ", légitimation, consécration, canonisation, comme on préférera ${ }^{43}$ et la patrimonialisation, différence dont il faut comprendre si elle est de degré ou de nature. Dans L'Institution littéraire de Jacques Dubois, la consécration «occupe une place clairement déterminée dans le processus de légitimation des œuvres et des auteurs, qui se laisse appréhender selon quatre étapes théoriques. La première est celle de l'émergence (vouloir-être de la littérature), qui est prise en charge par des instances de la vie littéraire telles que les salons, cénacles, écoles ou revues. La seconde correspond à la reconnaissance (être de la littérature) et est essentiellement assurée par les éditeurs. La troisième étape est spécifiquement celle de la consécration (être de la bonne littérature) et est le fait d'instances telles que la critique, les académies et les jurys. La quatrième et dernière phase du processus de légitimation est la canonisation (être un modèle de littérature, faire partie du patrimoine littéraire) et s'opère au sein de l'institution scolaire (programmes, manuels, dictionnaires des auteurs et des œuvres, anthologies, etc. $\left.{ }^{44}\right)$ ». Les étapes sont donc les mêmes que celles de la patrimonialisation selon la définition qu'en propose Guy Di Méo ${ }^{45}$.

Si pourtant la légitimation opère à l'intérieur du champ littéraire, la patrimonialisation échappe largement à ce champ. Du côté de la consécration, pour reprendre la liste des instances mentionnées par Dubois, naguère les salons et cénacles, aujourd'hui les écoles ou revues, les éditeurs, la critique, les académies et les jurys, l'institution scolaire dans son ensemble, plus spécifiquement l'Université; du côté de la patrimonialisation, l'école (primaire, éventuellement le collège), les sociétés savantes (versus l'Académie), les « petites » revues, revues de sociétés ou d'amis d'auteurs par exemple, les éditions grand public, les Maisons d'écrivains et avec elles les municipalités, les régions, etc. La patrimonialisation est l'indice d'un déplacement par lequel la reconnaissance "sort » du littéraire. Si Verne est devenu à Amiens un « objet patrimonial ", c'est bien sûr en tant qu'auteur des Voyages extraordinaires, c'est aussi en tant que personnalité locale, ancien conseiller municipal, grand ordonnateur de la construction du Cirque: un homme dont la principale vertu est d'avoir vécu à Amiens; aussi participe-t-il de la politique touristique de la ville à l'égal de la cathédrale, du macaron et de la ficelle picarde. Quand la légitimation vient de l'intérieur du champ, la patrimonialisation n'en procède pas nécessairement ou pas essentiellement. La différence est donc moins de degré que de nature.

Dans ce contexte, la patrimonialisation peut même devenir à l'occasion une sorte de "récupération » symbolique pour des écrivains ayant raté la légitimation. Ainsi Verne, qui rêvait d'être "écrivain ${ }^{46}$ ", c'est-à-dire reconnu pour son style et la qualité de ses descriptions ${ }^{47}$, ne parvint jamais à la consécration ${ }^{48}$. Il trône en revanche au panthéon des auteurs "du patrimoine». Légitimation et patrimonialisation semblent ainsi relever de deux gestes distincts pour deux publics distincts, l'un effectué dans le champ à destination de ses acteurs, l'autre hors du champ à destination d'un autre public sans doute, moins savant et plus large. J'ai dit plus haut la nature essentiellement politique du geste patrimonial. Elle se pose ici sous une forme légèrement différente: le 
« patrimoine littéraire » ne serait-il pas ce que «l'on » garde de la littérature pour les non-initiés?

\section{Conclusion}

31 La territorialisation impliquée par le processus patrimonial en est sans doute la principale pierre d'achoppement. Inscrivant l'écrivain dans un espace susceptible d'être valorisé en son nom, elle l'assigne en quelque sorte symboliquement à résidence, entraînant dans son sillage l'œuvre qui est la sienne : à rebours du "classique", universel, l'auteur «du patrimoine » occupe un lieu, habite un temps. Or s'il n'est pas question de nier toute référentialité, il convient en revanche de se souvenir que le texte, toujours, échappe à cette dimension entendue au sens strict: la littérature ne saurait être territorialisée sans risque de perdre l'universalité à laquelle elle prétend.

Rien n'est pourtant strictement figé, comme en témoigne le paradoxe sur lequel je voudrais terminer, patrimonialisation et universalité se rejoignant dans la définition juridique d'un "patrimoine littéraire européen ", reconnu en 2010 à l'occasion d'un arrêt rendu par la Cour européenne des droits de l'homme ${ }^{49}$. Elle a permis d'assurer avec succès la défense d'un éditeur, condamné en Turquie pour avoir publié les Onze mille verges d'Apollinaire: la notoriété internationale de l'auteur, la date déjà ancienne de la première parution du texte, sa publication dans de nombreux pays en diverses langues, sa diffusion sous forme de texte écrit et sur Internet, et, enfin, sa " consécration » par l'entrée dans la prestigieuse collection des éditions de La Pléiade, ont amené la Cour européenne des droits de l'homme à conclure que le public turc ne pouvait être privé d'accès à ce patrimoine. L'idée de " patrimoine littéraire européen » a pu ainsi contribuer à l'affaiblissement de la notion de "morale nationale", plus encore à la diffusion de la liberté d'expression : «La clef de cet éblouissant succès [...] est le concept inédit de patrimoine littéraire européen ${ }^{50}$.»

\section{NOTES}

1. L. Desgraves, Le patrimoine des bibliothèques: rapport à M. le directeur du livre et de la lecture, ministère de la Culture, 1982.

2. J.-P. Oddos (dir.), Le Patrimoine. Histoire, pratiques et perspectives, Paris, Éditions du Cercle de la librairie, coll. «Bibliothèques", 1997, cité par A. Marcetteau-Paul, dans «Le patrimoine, une valeur d'avenir », Bulletin des Bibliothèques de France, 2004, $\mathrm{n}^{\circ}$ 5, «Patrimoines », p. 35. En ligne : <http://bbf.enssib.fr/consulter/bbf-2004-05-0035-006> (consulté le 25 novembre 2018).

3. Voir à ce sujet l'exemple de la bibliothèque municipale de Douai présenté par P.-J. Lamblin, «Quand j'entends parler de patrimoine, je sors de ma réserve », ibid., p. 40-47. En ligne : <http:// bbf.enssib.fr/consulter/bbf-2004-05-0040-007> (consulté le 13 novembre 2018).

4. B. Louichon, «Le patrimoine littéraire : un enjeu de formation », Tréma, $n^{\circ} 43,2015$. En ligne : <http://trema.revues.org/3285> (consulté le 30 septembre 2016). DOI : 10.4000/trema.3285

5. Voir le texte de présentation du projet: < http://www.msh.univ-nantes.fr/34358274/0/ fiche__article/> (consulté le 13 novembre 2018). 
6. Un jeune historien, Thibault Le Hégarat, a ainsi récemment consacré un article à «Un historique de la notion de patrimoine » dans lequel il se propose «d'entreprendre un travail de compilation de définitions pour cerner la notion». En ligne: <https://halshs.archivesouvertes.fr/halshs-01232019> (consulté le 20 octobre 2018).

7. J.-P. Babelon et A. Chastel, La Notion de patrimoine, Paris, L. Lévy, 1994, p. 11.

8. C'est la première définition donnée par le Trésor de la langue française, rendant compte du sens du terme depuis le Moyen Âge. On peut ajouter qu'existe alors aussi le "matremoigne » pour désigner ce qui est hérité de la mère.

9. On est loin de la réalité de l'héritage familial, reçu en bloc, sans sélection aucune.

10. N. Heinich, La Fabrique du patrimoine. De la cathédrale à la petite cuillère, Paris, éditions de la Maison des Sciences de l'Homme, coll. « Ethnologie de la France », 2009.

11. A. Babadzan, «Les usages sociaux du patrimoine", Revue Ethnologie comparée, $\mathrm{n}^{\circ} 2$, " Miroirs identitaires », printemps 2001. En ligne: <https://lersem.www.univ-montp3.fr/fr/revuenum\%C3\%A9ro-2/les-usages-sociaux-du-patrimoine-alain-babadzan> (consulté le 20 octobre 2018).

12. H.-P. Jeudy, La Machinerie patrimoniale, Belval, Circé, coll. « Circé poche », 2008.

13. T. Le Hégarat, art. cité.

14. H.-P. Jeudy (dir.), Patrimoines en folie, Paris, éditions de la Maison des Sciences de l'Homme, coll. « Ethnologie de la France », 5, 1990.

15. Le «Référentiel AERES - Entités de recherche » de septembre 2015, non modifié lors de la transformation de l'AERES en HCERES, s'attache à définir les différences entre pluridisciplinarité, interdisciplinarité et transdisciplinarité. On lit ainsi p. 19: «La pluridisciplinarité est une juxtaposition de perspectives disciplinaires qui élargit le champ de la connaissance, en accroissant le nombre des données, des outils et des méthodes disponibles. Les composantes disciplinaires, dans ce cas, gardent leur identité. [...] /L'interdisciplinarité est la coopération de plusieurs disciplines autour de projets communs. [...] Les travaux en commun associent des données, des méthodes, des outils, des théories et des concepts issus de disciplines différentes en une synthèse dans laquelle le rôle des composantes disciplinaires va bien au-delà de la simple juxtaposition. [...] /La transdisciplinarité est une approche scientifique qui dépasse les points de vue disciplinaires par l'approche globale d'une question. Elle témoigne d'un degré d'intégration supplémentaire par rapport à l'interdisciplinarité, que les disciplines partenaires atteignent lorsque cette pratique répétée débouche sur la définition de nouveaux paradigmes et sur la formation d'une communauté qui les partage, faisant ainsi émerger peu à peu une nouvelle discipline. "

16. L'expression apparaît par exemple dans la présentation en ligne du LabEx PATRIMA, université Paris-Saclay: <https://www.universite-paris-saclay.fr/fr/recherche/projet/labexpatrima> (consulté le 13 novembre 2018).

17. Voir <https://ich.unesco.org/fr/qu-est-ce-que-le-patrimoine-culturel-immateriel-00003>, consulté le 20 octobre 2018 ; je souligne. Sur cette question, voir É. Fagnoni, « Patrimoine versus mondialisation?», Revue Géographique de l'Est, vol.53, nº 3-4, 2013. En ligne: <http:// rge.revues.org/5048> (consulté le 22 octobre 2018).

18. Sur cette question, voir notamment G. Di Méo, «Processus de patrimonialisation et construction des territoires ", communication prononcée au colloque Patrimoine et industrie en Poitou-Charentes : connaitre pour valoriser, Poitiers-Châtellerault, septembre 2007, La Crèche, Geste éditions, 2008, p. 87-109. En ligne : <halshs-00281934>.

19. Ibid.

20. Il est tout à fait symptomatique à cet égard que la mission de patrimonialisation inscrite dans les objectifs premiers de la Fondation du patrimoine ait été confiée à Stéphane Bern, raconteur d'histoire(s). 
21. «Il appert, en premier lieu, que la création patrimoniale se dessine, s'accélère et se diversifie principalement dans le cours ou à l'issue de crises sociales intenses [...]. / Ainsi, la notion classique et monumentale de patrimoine a émergé, dans les sociétés occidentales, à la suite de révolutions bourgeoises: après Cromwell, à la fin du XVII siècle, en Angleterre; après la Révolution, en France, au xIx ${ }^{\mathrm{e}}$ siècle. [...] / Autre traumatisme collectif, la Deuxième Guerre mondiale et ses destructions massives d'espaces industriels ont conduit l'Angleterre des années 1950 à développer une représentation patrimoniale très précoce de son tissu industriel. / Le patrimoine en plein développement des campagnes et de la ruralité [...] s'édifie, dans la société d'aujourd'hui, sur les vestiges d'une société paysanne et agricole en perdition. Finalement, sans crise rurale point de patrimoine du même nom, serait-on tenté d'affirmer ! Par ailleurs, n'est-ce pas une réelle peur alimentaire [...] qui ont [sic] conféré à la consommation de produits du terroir, de plus en plus patrimonialisés (AOC, IGP, AS), une forte croissance ?", G. Di Méo, art. cité.

22. B. Louichon, art. cité.

23. Ibid.

24. Je pousse volontairement les choses à leur limite, en introduisant un biais dont j'ai parfaitement conscience : les biens architecturaux ne figurent pas sur la même « liste » que les boissons ou autres fromages; il n'en reste pas moins que le même terme de "patrimoine " s'impose.

25. B. Louichon, art. cité.

26. L'Assemblée générale constitutive de la Fédération des maisons d'écrivain et des patrimoines littéraires s'est tenue le 6 décembre 1997.

27. 200 établissements scolaires portaient en 2010 le nom de Verne, si l'on en croit Claude Lelièvre, dans un article publié sur le blog de Médiapart, « Du nom des écoles ». En ligne : <https://blogs.mediapart.fr/claude-lelievre/blog/271210/du-nom-des-ecoles> (consulté le 13 novembre 2018), loin derrière Prévert (407) et Hugo (337).

28. C. Baron, «Littérature et géographie : lieux, espaces, paysages et écritures ", Fabula-LhT, n ${ }^{\circ} 8$, «Le Partage des disciplines», mai 2011. En ligne: <http://www.fabula.org/lht/8/baron.html> (consulté le 7 novembre 2018).

29. M. Collot, «Pour une géographie littéraire ", Fabula-LhT, $\mathrm{n}^{\circ} 8$, « Le Partage des disciplines », mai 2011. En ligne : <http://www.fabula.org/lht/8/collot.html> (consultée le 7 novembre 2018).

30. L'article de Jean-Paul Dekiss consacré à la Maison de Jules Verne montre clairement l'ampleur de son ambition lorsqu'il fut chargé d'aménager puis de valoriser la maison de Jules Verne à Amiens : J.-P. Dekiss, « La Maison d'un écrivain, utopie ou enjeu de société », RHLF, vol. 109, $\mathrm{n}^{\circ} 4$, 2009, p. 783-795.

31. M. Collot, art. cité.

32. "Il est des lieux où souffle l'esprit ", écrivait Barrès aux premières lignes de La Colline inspirée. 33. D. Fabre, « Maison d'écrivain. L'auteur et ses lieux », Le Débat, vol. 3, nº 115, 2011, p. 172-177, ici p. 175.

34. Ibid.

35. D. Saurier, "Proust dans ses meubles. Patrimonialisation de la Maison de tante Léonie ", Ethnologie française, $\mathrm{n}^{\circ}$ 3, vol. 37, 2007, p. 541-549, ici p. 547.

36. Ibid., p. 548.

37. Voir le site de la Lorraine des écrivains : <http://lalorrainedesecrivains.univ-lorraine.fr/ cartographie-patrimoine-litteraire/> (consulté le 15 novembre 2018).

38. D. Fabre, art. cité.

39. J.-C. Bailly, Le Dépaysement. Voyages en France, Paris, Seuil, coll. «Fiction \& Cie », 2011. Voir également «L'Europe des écrivains. La France de Christine Angot, Jean-Christophe Bailly et Marie Darrieussecq », diffusé le 11 novembre 2015, Institut national de l'audiovisuel, <http:// 
catalogue-productions.ina.fr/art-culture/leurope-des-ecrivains-la-france-de-christine-angotjean-christophe-bailly-et-marie-darrieussecq/> (consulté le 21 novembre 2018).

40. On peut à cet égard saluer certains travaux, comme le web-documentaire réalisé par le Centre d'études des relations et des contacts linguistiques et littéraires de l'université de Picardie sous le titre "Ancrages Passages»: consacré à "Sept écrivains en Picardie», il ne se contente pas d'étudier leur ancrage, résiste à la tentation de les territorialiser pour, au contraire, insister sur les «passages» qu'ils promeuvent ou suggèrent. Voir: <http://canalnord.org/ancragespassages/\#ACCUEIL> (consulté le 25 novembre 2018).

41. L'expression, élaborée par Jean-Noël Retière, s'est imposée dans les travaux de sociologie : voir notamment É. Aunis, J. Benet, A. Mège et I. Prat (dir.), Les Territoires de l'autochtonie, Rennes, PUR, 2016.

42. C. CHAULET-ACHOUR, "Patrimoine littéraire et écrivaines francophones", Le français aujourd'hui, vol.4, no 163, 2008, p.7-15. DOI : 10.3917/lfa.163.0007. En ligne: <https:// www.cairn.info/revue-le-francais-aujourd-hui-2008-4-page-7.htm> (consulté le 25 novembre 2018) : "Revenons maintenant à la question du patrimoine. Le patrimoine induit héritage et transmission. Cela oblige lorsqu'on veut choisir un corpus pour des programmes de littérature, à ne pas céder au coup de cœur du dernier roman ou du dernier recueil de poèmes mais à s'appuyer sur des œuvres qui ont déjà acquis patine et légitimité. [...] / Aussi ce corpus littéraire me semble devoir, pour entrer dans les programmes avec quelque crédibilité, être circonscrit aux écrivaines devenues « classiques » et dont on ne peut faire l'économie [...] 》 (je souligne).

43. Ce n'est pas le lieu d'entrer dans les détails de ce qui pourrait distinguer légitimation et consécration, consécration et canonisation, non plus que de trancher finement entre la nature du « canon » et celle du corpus légitimé par exemple. Il s'agit simplement d'essayer de comprendre si la patrimonialisation est instance de légitimation - c'est le terme que j'emploierai de préférence - à l'égal de la consécration ou de la canonisation.

44. Je reprends la lecture que propose Benoît Denis dans "La consécration ", COnTEXTES, $\mathrm{n}^{\circ} 7$, 2010. En ligne [3 juin 2010]: <http://journals.openedition.org/contextes/4639> (consulté le 25 octobre 2018). DOI : $10.4000 /$ contextes.4639

45. Voir supra, art. cité.

46. On relira par exemple sa lettre à Pierre-Jules Hetzel, le 25 avril 1864, alors qu'il se lance tout juste dans la rédaction des Voyages extraordinaires: "D'ailleurs, je vais vous dévoiler toute ma pensée, mon cher Hetzel; je ne tiens pas énormément à être un arrangeur de faits; par conséquent, je serai toujours prêt à modifier pour le bien général. $\underline{\text { Ce que je voudrais devenir }}$ avant tout, c'est un écrivain, louable ambition que vous approuverez pleinement.», Correspondance inédite de Jules Verne et de Pierre-Jules Hetzel (1863-1886), établie par O. Dumas, P. Gondolo della Riva et V. Dehs, t. I (1863-1874), Genève, Slatkine, 1999, p. 28 (je souligne).

47. Ibid., un peu plus loin dans la lettre: "Vous me dites des choses bien aimables et bien flatteuses sur mon style qui s'améliore. Évidemment, vous devez faire allusion aux passages descriptifs dans lesquels je me déploie de mon mieux [...]».

48. Sa reconnaissance en tant qu'écrivain à part entière date des années 1960 et on peut considérer qu'elle n'est pas encore totalement accomplie : en témoigne, entre autres choses, son absence dans les programmes de l'agrégation de lettres, instance de légitimation s'il en est. Il n'y intervient qu'au titre des programmes de littérature comparée, comme s'il n'était pas digne d'y figurer «à part entière ». Dans le même esprit, on peut souligner qu'il n'a pas les honneurs des " Euvres complètes" de la Bibliothèque de La Pléiade, dans laquelle ne sont publiés que quelques-uns de ses romans, rassemblés en quatre volumes; et encore cette publication est-elle très récente (2012, 2016 et 2017).

49. Il s'agit de l'arrêt de chambre Akdas c/ Turquie du 16 février 2010. 
50. Voir J.-P. Marguénaud et B. Dauchez, «Les Onze mille verges fondatrices du patrimoine littéraire européen », Recueil Dalloz, Dalloz, 2010, p. 1051. <https://hal.archives-ouvertes.fr/ hal-00652544/>

\section{RÉSUMÉS}

La littérature a récemment été inscrite au «patrimoine » avec l'introduction du socle commun des connaissances dans les programmes scolaires entre 2008 et 2012. Favorisée par l'indétermination du concept, son caractère transdisciplinaire, sa dimension consensuelle et les perspectives économiques et commerciales qu'il ouvre, cette patrimonialisation n'est pas sans conséquences sur la littérature. Elle contribue à la désigner comme un objet en péril, menacé de disparition. Surtout, elle la territorialise, la détachant ainsi de l'universalité dont se réclamait le canon. À rebours de la légitimation, la patrimonialisation se joue pour l'essentiel à l'extérieur du champ littéraire, définissant, à côté de la culture savante une sorte de culture populaire orchestrée par un grand récit national.

Literature has recently been listed as part of "heritage" as a result of the introduction of the "common base of knowledge" in school curricula between 2008 and 2012. Because of the indeterminacy of the concept, of its transdisciplinary nature, of its consensual dimension and of the economic and commercial prospects it opens, this patrimonialization is not without consequences for literature. It contributes to its identification as endangered object. Above all, it territorializes-literature, untiying it from the universality advocated by the canon. Against legitimation, patrimonialization is mostly at work outside the literary field, defining, alongside high culture, a form of popular culture orchestrated by a great national narrative.

\section{INDEX}

Mots-clés : patrimonialisation, territorialisation, légitimation, récit national

Keywords : patrimonialization, heritage, territorialization, legitimation, national narrative

\section{AUTEUR}

\section{MARIE-FRANÇOISE MELMOUX-MONTAUBIN}

Ancienne élève de l'ENS, agrégée de Lettres classiques, Marie-Françoise Melmoux-Montaubin est professeur de littérature française à l'université de Picardie Jules Verne. Spécialiste du roman de la seconde moitié du XIX ${ }^{\mathrm{e}}$ siècle, elle travaille depuis plusieurs années sur les relations entre presse et littérature et a récemment édité les premières chroniques de Maupassant (Garnier, 2019). Présidente du Centre international Jules Verne, elle s'intéresse beaucoup à l'œuvre du romancier et à sa réception. 